\title{
Clinical patterns of familial inflammatory bowel disease
}

\author{
J Satsangi, C Grootscholten, H Holt, D P Jewell
}

\begin{abstract}
Background-Although many recent studies have shown the increased risk of inflammatory bowel disease in relatives of patients with Crohn's disease and ulcerative colitis, clinical patterns of disease within families remain relatively poorly documented.

Aims-In this study, clinical characteristics (disease type, extent, age on onset, need for surgery, and presence of extraintestinal manifestations) have been compared in affected subjects in multiplyaffected families, with inflammatory bowel disease.

Methods-54 families in whom one parent and at least one child were affected (a total of 77 parent-child pairs) and 155 families in whom at least two siblings were affected (a total of 190 affected sibling pairs) were involved.

Results-In affected parent-child pairs, parent and child were concordant for disease type in 58 of 77 pairs $(75 \cdot 3 \%$ ), for extent in $63.6 \%$, extraintestinal manifestations in $70.1 \%$, and smoking history in $85 \%$. The median age of onset in parents was significantly higher than offspring ( $<<0.0001$ ). In 40 pairs, 60.6\%, the parent was at least 10 years older than child. Siblings were concordant for disease type in $81 \cdot 6 \%$ of the affected sibling pairs, extent in $76.0 \%$, extraintestinal manifestations in $83.8 \%$, and smoking history in $81 \cdot 3 \%$. In contrast with the parent-child pairs, 68.1\% (111 sibling pairs) siblings were diagnosed within 10 years of each other. The median age of onset was $24 \cdot 0$ years.

Conclusions-This study has shown consistent clinical patterns in many families with inflammatory bowel disease. The differences in age of onset between parents and children are not readily explained by a simple cohort effect or ascertainment bias, and may reflect effects of genetic factors, producing anticipation between generations.
\end{abstract}

Gastroenterology Unit, Radcliffe Infirmary, Oxford

J Satsangi

C Grootscholten

H Holt

D P Jewell

Correspondence to: Dr J Satsangi,

Gastroenterology Unit,

Radcliffe Infirmary,

Woodstock Road, Oxford

WX2 6HE.

Accepted for publication

29 December 1995

Keywords: ulcerative colitis, Crohn's disease, inflammatory bowel disease, genetic participation.

Many recent studies have shown the increased prevalence of inflammatory bowel disease among relatives of patients with Crohn's disease and ulcerative colitis. ${ }^{1-3}$ First degree relatives are at greatest risk, particularly siblings, but more distant relatives may also be affected. Both genetic and environmental factors seem to be pertinent to familial disease: comparison of concordance rates in monozygotic and dizygotic twins suggests that genetic factors may be relatively more important in the pathogenesis of Crohn's disease than ulcerative colitis, or indeed than in a number of other common disorders. ${ }^{4}$ However, no simple Mendelian pattern of inheritance is evident for either Crohn's disease or ulcerative colitis and it seems most likely that inflammatory bowel disease' represents a heterogeneous group of diseases of polygenic inheritance. The extent of the heterogeneity, the number and identity of susceptibility genes, and the relation between genotype and disease phenotype, remain uncertain. ${ }^{5}$

Relatively little attention has been paid to defining the clinical patterns of familial inflammatory bowel disease. Such data may be of considerable value in understanding the extent of disease heterogeneity, and the relative effects of genetic and environmental factors. Recent data from studies in France ${ }^{6}$ and the United States ${ }^{7}$ have shown noticeable concordance in members of multiply affected families with Crohn's disease extent and behaviour; the authors have suggested that familial phenotypes reflect genetically determined differences.

In this study, the clinical characteristics of inflammatory bowel disease in members of 192 multiply affected families have been compared.

\section{Methods}

\section{Subjects}

One hundred and ninety two families in whom two or more first degree relatives have inflammatory bowel disease were identified between January 1993 and December 1994, with the help of gastroenterologists throughout the United Kingdom and the National Association for Crohn's and Colitis. Only north European white subjects were studied to exclude any effects of ethnic differences.

In 54 families, at least one parent and one child were affected - a total of 77 parent-child pairs were available.

In 155 families, at least two siblings were affected - a total of 190 affected sibling pairs.

In 17 families, one parent and two siblings

\section{Clinical data}

Clinical details were obtained from each family member (by questionnaire and personal interview, where possible) and from the hospital clinician involved, who was asked to provide 
clinical, radiological, and endoscopic information. Case notes were also obtained if practicable and, for each subject, the following data were collected: sex, age of onset of symptoms, disease type (Crohn's disease, ulcerative colitis or indeterminate colitis); disease extent, presence of extraintestinal manifestations (erythema nodosum, pyoderma gangrenosum, iritis, uveitis, arthritis), need for surgery, or smoking habit at diagnosis. Each subject was simply classified as either a smoker or a nonsmoker.

Disease extent was assessed and classified on the basis of clinical data obtained. For Crohn's disease, the extent was classified as small intestinal, colonic or ileocolonic (including terminal ileal disease). For ulcerative colitis, extent was classified as 'distal' or 'extensive' relative to the splenic flexure, by the authors on the basis of information provided by the hospital physician involved. If the macroscopic assessment of disease extent was different from the disease extent assessed by histological examination, the authors relied on the histological data. For each patient, the imaging technique used (colonoscopy or barium enema) depended on the clinical practice of the physician involved. Thus in a given sibling pair, different imaging methods may have been used for each relative. If results of recent imaging were not available, or if the disease type seemed uncertain from the data provided, the family was excluded from analysis.

\section{Statistics}

Concordance for clinical characteristics between different groups was compared using contingency tables, and $\chi^{2}$ statistics.

\section{Results}

\section{Parent-child pairs}

Seventy seven affected parent-child pairs were studied. Of the affected parents, 30 were male and 47 female. Thirty nine parents had Crohn's disease, 37 ulcerative colitis, and only one indeterminate colitis. Of the offspring, 42 had Crohn's disease and 35 ulcerative colitis.

Fifty eight parent-child pairs were concordant for disease type (27 concordant for ulcerative colitis; 31 for Crohn's disease). Table I shows the concordance rates for each clinical characteristic in these parent-child pairs.

Differences in smoking habits between parent and child were compared with concordance rates for disease extent, extraintestinal manifestations, and need for surgery: no consistent pattern was evident.

\section{Age of onset}

In parent-child pairs, the median age of onset for affected parents was 38.0 years, compared with 22.0 for their affected offspring $\left(\chi^{2}<800\right.$, $\mathrm{p}<110^{-6}$ ). In $78 \cdot 8 \%$ of pairs, the parent was more than five years older than the child at onset (more than 10 years in $60.4 \%$ ). In only
TABLE I Concordance rate (\%) for clinical characteristics in affected parent-child pairs

\begin{tabular}{|c|c|c|c|}
\hline & $\begin{array}{l}\text { All pairs } \\
(n=77)\end{array}$ & $\begin{array}{l}C D-C D^{\star} \\
\text { pairs } \\
(n=31)\end{array}$ & $\begin{array}{l}U C-U C+ \\
\text { pairs } \\
(n=27)\end{array}$ \\
\hline $\begin{array}{l}\text { Sex } \\
\text { Age of onset } \\
\text { Disease type } \\
\text { Disease extent } \S \\
\text { Extraintestinal manifestations } \\
\text { Need for surgery\| } \\
\text { Smoking habit at diagnosis }\end{array}$ & $\begin{array}{l}55 \cdot 8 \\
39 \cdot 6 \\
75 \cdot 3 \\
63 \cdot 6 \\
70 \cdot 1 \\
56 \cdot 6 \\
85 \cdot 0\end{array}$ & $\begin{array}{c}54 \cdot 8 \\
42 \cdot 7 \\
100 \\
75 \\
68 \cdot 0 \\
37 \cdot 0 \\
76 \cdot 9\end{array}$ & $\begin{array}{c}63 \cdot 0 \\
32 \cdot 0 \\
100 \\
53 \\
73 \cdot 9 \\
76 \cdot 9 \\
95 \cdot 2\end{array}$ \\
\hline
\end{tabular}

*Both parent and child having Crohn's disease. †Both parent and child having ulcerative colitis. $¥$ Relatives were considered concordant if the difference in age of onset was less than 10 concordant if the difference in age of onset was less than 10
years. SComplete data were only available for 17 UC-UC and 16 CD-CD pairs. \|Relatives were considered concordant if both had undergone surgical resection of bowel for inflammatory bowel disease.

seven cases was the child older than the parent at onset of symptoms. The calendar years of onset for parent and offspring were discordant, effectively ruling out the possibility that these data have arisen as a result of simultaneous exposure to a shared environmental pathogen.

\section{Affected sibling pairs}

One hundred and ninety pairs of affected siblings were included. Of these 380 patients, 196 had Crohn's disease, 169 ulcerative colitis, and 15 indeterminate colitis. In 82 pairs, both siblings had Crohn's disease, and in 69 both had ulcerative colitis. Table II shows the concordance rates for sex, age of onset, disease extent, extraintestinal manifestations, and need for surgery. In siblings concordant for disease, smoking habits were compared with concordance for other characteristics: no consistent pattern with regard to disease extent, need for surgery or extraintestinal manifestations was noted.

\section{Twin pairs}

Eleven twin pairs were identified (two monozygotic, three dizygotic, and six of uncertain zygosity).

In eight twin pairs, both twins were affected. Seven were definitely concordant for disease type: of these six were concordant for extent, age of onset, extraintestinal manifestations, and smoking history.

The twins discordant for disease type were male, of uncertain zygosity. One was diagnosed as having ileocolonic Crohn's disease at

TABLE II Concordance rate (\%) for clinical characteristics in affected sibling pairs

\begin{tabular}{llcc}
\hline & $\begin{array}{c}\text { All pairs } \\
(n=190)\end{array}$ & $\begin{array}{l}C D-C D^{\star} \\
\text { pairs } \\
(n=82)\end{array}$ & $\begin{array}{l}\text { UC-UC† } \\
\text { pairs } \\
(n=69)\end{array}$ \\
\hline Sex & $62 \cdot 0$ & $61 \cdot 0$ & $65 \cdot 2$ \\
Age of onset $\ddagger$ & $68 \cdot 1$ & $78 \cdot 9$ & $62 \cdot 5$ \\
Disease type & $81 \cdot 6$ & 100 & 100 \\
Disease extent $\$$ & $76 \cdot 0$ & $80 \cdot 3$ & $68 \cdot 6$ \\
Extraintestinal manifestations & $83 \cdot 8$ & $80 \cdot 3$ & $88 \cdot 9$ \\
Need for surgery\| & $60 \cdot 7$ & $56 \cdot 0$ & $66 \cdot 7$ \\
Smoking habit at diagnosis & $81 \cdot 3$ & $78 \cdot 4$ & $82 \cdot 5$ \\
\hline
\end{tabular}

^Both relatives had Crohn's disease. †Both relatives had ulcerative colitis. $¥$ Siblings were considered concordant if the difference in age of onset was less than 10 years. \$Complete data were only available for $61 \mathrm{CD}-\mathrm{CD}$ and 35 UC-UC pairs. |lRelatives were considered concordant if both had undergone resection of bowel for inflammatory bowel disease. 
36 years of age, requiring surgery. His brother developed proctosigmoiditis 12 months later. Small bowel enema was normal. Colonic histology strongly favours ulcerative colitis over Crohn's disease of the colon.

\section{Discussion}

These data have been collected in the course of a detailed study of the genetics of inflammatory bowel disease currently in progress. More than 1500 family members and 500 clinicians have taken part. Considerable efforts have been made, not only to collate all available clinical information, but to verify these data with particular respect to disease type and extent. Evidence has emerged for a number of consistent clinical patterns of familial disease of potential significance in the pathogenesis of inflammatory bowel disorders.

In all pairs of affected relatives, a high degree of concordance for disease type was noted - of 240 relative pairs, 185 were concordant for disease type. These data are in keeping with the results of many previous studies of familial inflammatory bowel disease, including the authors' recent study. ${ }^{1}$ Relatives of patients with Crohn's disease, and ulcerative colitis are at increased risk of inflammatory bowel disease, particularly the concordant disease type.

Moreover, within pairs of relatives concordant for Crohn's disease, a high degree of concordance was noted for the phenotypic characteristics of disease, namely disease extent and extraintestinal manifestations. These data complement the reported observations of investigators in the United States ${ }^{7}$ and France $^{6}$. Concordance for disease extent in 35 affected relative pairs with Crohn's disease was described by clinicians at the Johns Hopkins Medical School. Subsequently similarities in disease extent and behaviour (transmural aggressiveness) were noted in 69 French families.

This study is the first to compare clinical characteristics in affected relatives with ulcerative colitis within pairs of relatives concordant for ulcerative colitis. Although high concordance for the presence of extraintestinal manifestations was observed, it was noticeable that only $53 \%$ of affected parent-child pairs, and $68 \%$ of sibling pairs were concordant for extent of colonic involvement. The discordance did not reflect differences in smoking habits between the affected relatives. In fact, relative pairs were remarkably concordant for smoking habit.

Seven of eight pairs of affected twins identified in this study were definitely concordant for disease, and six of these were strongly concordant for clinical characteristics. However, the other pair appear discordant: further studies are underway to determine zygosity and to confirm paternity.

There are many well recognised difficulties in attempting to classify either Crohn's disease or ulcerative colitis by 'phenotype'. ${ }^{8}$ Disease extent, severity, and behaviour (fistulising or stenotic for Crohn's disease) have all been suggested, ${ }^{9}$ but are yet to be evaluated in a prospective study. Indeed, even in ulcerative colitis where the distinction between distal and extensive disease is used in clinical practice, evidence is emerging that disease extent is subject to considerable variation over time and may not be useful as stable phenotypic characteristic. ${ }^{10}$

These considerations notwithstanding, the clinical patterns shown in this study provide further evidence for genetic heterogeneity: according to this model, inflammatory bowel disease may be regarded as a heterogeneous group of related diseases, each of multifactorial aetiology. Although predisposing factors (genetic or environmental) may be shared between sub-groups, and account for 'mixed disease families', the combination of susceptibility factors differentiates different subgroups. At present, the extent of heterogeneity, and relation between genotype and phenotype and the number of, or identity of genes involved, is uncertain. However, immunogenetic studies from Japan, ${ }^{11}$ California, ${ }^{12}$ and Western Europe ${ }^{13-15}$ have all recently provided evidence for heterogeneity. Association studies and linkage analysis have implicated HLA class II genes as important determinants of both phenotype and susceptibility in ulcerative colitis, but of less definite importance in Crohn's disease. Other non-HLA genes have also been suggested as determinants of extent and severity. ${ }^{16}$

Of particular interest in this study are the differences in age of onset between affected parents and their children. Polito and colleagues have presented similar data concerning 27 parent-child pairs - the children were not only significantly younger at age of onset of disease, but suffered more severe disease. ${ }^{7}$

The explanation for this phenomenon is uncertain. A simple cohort effect due to simultaneous exposure to environmental pathogen was not apparent: the calendar year of diagnosis in parent and child were discordant. Simple reporting bias also seems unlikely - if symptomatic children were presented to physicians at an early age, simply because of anxiety regarding another member of the family, then discordance for age of onset might also be expected in sibling pairs.

Genetic anticipation, the tendency for successive generations to develop disease of increasing severity and earlier onset - has been observed in monogenic disorders, including Huntingdon's disease and the Fragile $\mathrm{X}$ syndrome, and has recently been suggested to be relevant to polygenic disease, notably rheumatoid disease and schizophrenia. ${ }^{17} 18$ The molecular basis for this phenomenon involves the progressive amplification of unstable triplets repeats of DNA. These contribute to DNA instability.

There is now great interest in identifying susceptibility genes in Crohn's disease and ulcerative colitis. Advances in semi-automated DNA technology are such that a systematic search involving the whole human genome for susceptibility genes is practicable. ${ }^{19}$ This is underway in a number of centres. The results 
are awaited with considerable interest and may have great significance as regards disease pathogenesis. The clinical findings reported in this study add impetus to these investigations.

The authors are grateful to the families who have taken part in the study and the clinicians who have provided clinical data. Jack Satsangi is a Medical Research Council Training Fellow. Heather Holt is supported by Astra Draco.

1 Satsangi J, Rosenberg WMC, Jewell DP. The prevalence of inflammatory bowel disease in relatives of patients with inflammatory bowel disease in relatives of patients with Crohn's disease. European four

2 Orholm M, Munkholm P, Langholz E, Haagen Nielsen O, Sorensen TIA, Binder V. Familial occurrence of inflammatory bowel disease. N Engl f Med 1991; 324: 84-8.

3 McConnell RB, Vadheim CM. Inflammatory bowel disease. In: King RA, Rotter JI, Motulsky AO, eds. The genetic basis of common diseases. Oxford: Oxford University Press, 1992. 326-48.

4 Tysk C, Lindberg E, Järnerot G, Flodérus-Myrhed B. Ulcerative colitis and Crohn's disease in an unselected population of monozygotic and dizygotic twins. A study of population of monozygotic and dizygotic twins. A study of 990-6.

5 Satsangi J, Jewell DP, Rosenberg WMC, Bell JI. Genetics of inflammatory bowel disease. Gut 1994; 35: 696-700.

6 Gower-Rousseau C, Plegat S, Evrard JP, Grandbastien B, Hugot JP, Gendre JP. Familiarity in clinical characteristics of Crohn's disease in 69 French families. Gastroenterology 1995; 108: A826.

7 Polito J, Tokayer A, Childs B, Harris M, Bayless T. Crohn's disease: evidence for genetic heterogeneity and 'anticipation'. Gastroenterology 1995; 108: A503.

8 Satsangi J, Jewell DP. Genetics of inflammatory bowel disease. In: Proceedings of Falk Symposium No 85. disease. In: Proceedings of Falk
9 Sachar DB, Andrews HA, Farmer RG, L'Pallone F, Pena AS, Prantera C, et al. Proposed classification of patient subgroups in Crohn's disease. Gastroenterology Internubgroups in Crohn's

10 Langholz E, Nielsen OH, Munkholm P, Davidsen M Binder V. Course and prognostic factors influencing anatomical extent of ulcerative colitis. Gastroenterology 1995; 108: A857.

11 Kobayashi K, Atoh M, Konoeda Y, Yagita A, Inoko $\mathrm{H}$, Sekiguchi S. HLA-DR, DQ and T cell antigen recepto constant beta genes in Japanese patients with ulcerative colitis. Clin Exp Immunol 1990; 80: 400-3.

12 Toyoda H, Wang S-J, Yang H, Redford A, Magalong D, Tyan D. Distinct association of HLA class II genes with inflammatory bowel disease. Gastroenterology 1993; 104: 741-8.

13 Satsangi J, Welsh KI, Bunce M, Bell JI, Jewell DP. HLA Class II genes are important determinants of susceptibility in ulcerative colitis, but not in Crohn's disease. Gut 1995; 37 (suppl 2): A21

14 Satsangi J, Welsh KI, Bunce M, Julien C, Farrant JM, Bell JI, et al. Contribution of genes of the major histocompatibility complex to susceptibility and disease phenotype in inflammatory bowel disease. Lancet (in press).

15 Wassmuth R, Keller Y, Thomson G, Starck M, Lindhagen T, Holmberg E. HLA DRB1 alleles provide protection against Crohn's disease in Caucasians. European fournal of Gastroenterology and Hepatology 1994; 6: 405-11.

16 Mansfield JC, Holden H, Tarlow JK, Di Giovine FS, McDowell TL, Wilson AG, et al. Novel genetic association between ulcerative colitis and the anti-inflammatory cytokine interleukin-1 receptor antagonist. Gastroenterology 1994; 106: 637-42.

17 Petronis A, Kennedy JL. Unstable genes - unstable mind? Am $₹$ Psychiatry 1995; 152: 164-72.

18 McGuffin P, Owen MJ, Farmer AE. Genetic basis of schizophrenia. Lancet 1995; 346: 678-2.

19 Weissenbach J, Gyapay G, Dib C, Vignal A, Morisette J, Millasseau $\mathrm{P}$, et al. A second generation linkage map of the human genome. Nature 1992; 359: 794-801. 